University of New Orleans

ScholarWorks@UNO

10-13-2011

\title{
Three-dimensional polarization states of monochromatic light fields
}

R. M.A. Azzam

University of New Orleans, razzam@uno.edu

Follow this and additional works at: https://scholarworks.uno.edu/ee_facpubs

Part of the Electrical and Electronics Commons, and the Optics Commons

\section{Recommended Citation}

R. M. A. Azzam, "Three-dimensional polarization states of monochromatic light fields," J. Opt. Soc. Am. A $28,2279-2283(2011)$

This Article is brought to you for free and open access by the Department of Electrical Engineering at ScholarWorks@UNO. It has been accepted for inclusion in Electrical Engineering Faculty Publications by an authorized administrator of ScholarWorks@UNO. For more information, please contact scholarworks@uno.edu. 


\title{
Three-dimensional polarization states of monochromatic light fields
}

\author{
R. M. A. Azzam \\ Department of Electrical Engineering, University of New Orleans, New Orleans, Louisiana 70148, USA \\ (razzam@uno.edu)
}

Received July 14, 2011; revised September 14, 2011; accepted September 16, 2011; posted September 16, 2011 (Doc. ID 150996); published October 13, 2011

\begin{abstract}
The $3 \times 1$ generalized Jones vectors (GJVs) $\left[\begin{array}{lll}E_{x} & E_{y} & E_{z}\end{array}\right]^{t}(t$ indicates the transpose) that describe the linear, circular, and elliptical polarization states of an arbitrary three-dimensional (3-D) monochromatic light field are determined in terms of the geometrical parameters of the 3-D vibration of the time-harmonic electric field. In three dimensions, there are as many distinct linear polarization states as there are points on the surface of a hemisphere, and the number of distinct 3-D circular polarization states equals that of all two-dimensional (2-D) polarization states on the Poincaré sphere, of which only two are circular states. The subset of 3-D polarization states that results from the superposition of three mutually orthogonal $x, y$, and $z$ field components of equal amplitude is considered as a function of their relative phases. Interesting contours of equal ellipticity and equal inclination of the normal to the polarization ellipse with respect to the $x$ axis are obtained in 2-D phase space. Finally, the $3 \times 3$ generalized Jones calculus, in which elastic scattering (e.g., by a nano-object in the near field) is characterized by the 3-D linear transformation $\boldsymbol{E}_{s}=\boldsymbol{T} \boldsymbol{E}_{i}$, is briefly introduced. In such a matrix transformation, $\boldsymbol{E}_{i}$ and $\boldsymbol{E}_{s}$ are the $3 \times 1$ GJVs of the incident and scattered waves and $\boldsymbol{T}$ is the $3 \times 3$ generalized Jones matrix of the scatterer at a given frequency and for given directions of incidence and scattering. (c) 2011 Optical Society of America

OCIS codes: $\quad 260.0260,260.5430,260.6970,350.7420$.
\end{abstract}

\section{INTRODUCTION}

A monochromatic light beam traveling in the $z$ direction in free space or a homogeneous optically isotropic medium is essentially transverse electromagnetic $\left(E_{z} \approx 0\right)$ if the beam diameter $D$ (a measure of its lateral confinement in the $x y$ plane) is $\gg$ the wavelength $\lambda$ (see, e.g., [1] ). The polarization of such a beam is described by a $2 \times 1$ Jones vector $\left[\begin{array}{ll}E_{x} & E_{y}\end{array}\right]^{t}$ ( $t$ indicates the transpose) whose elements $E_{x}$ and $E_{y}$ are complex numbers or phasors that represent the oscillating mutually orthogonal $x$ and $y$ transverse components of the electric field $\vec{E}$. More concisely, the state of polarization is represented by the complex number $\chi=E_{y} / E_{x}$ or the corresponding point on the Poincaré sphere that is related to the complex $\chi$ plane by a stereographic projection [2, 3 ] .

However, if a Gaussian laser beam is brought to a narrow focus by a lens of high numerical aperture, the beam diameter $D$ in the focal region becomes comparable to $\lambda$ and the electric field can no longer be considered transverse or two dimensional. Furthermore, if the light beam incident on the convergent lens is apodized and radially polarized, the longitudinal component $E_{z}$ can become dominant $[\underline{4}, \underline{5}]$.

Three-dimensional (3-D) polarization states (PSs) are also encountered in transverse-magnetic guided waves [6], evanescent waves [ $[7,8]$, and in near-field and nano-optics $[\underline{7}, 9]$. It is apparent that 3 -D PSs have to be described by $3 \times 1$ generalized Jones vectors (GJVs): $\left[\begin{array}{lll}E_{x} & E_{y} & E_{z}\end{array}\right]^{t}$.

For a time-harmonic or monochromatic light field of given angular frequency $\omega$, to which this paper is confined, the locus of the tip of the electric vector at any point in space is in general elliptic $[\underline{3}, \underline{10}]$. Statistical or coherence properties of $3-\mathrm{D}$ PSs of quasi-monochromatic light fields have been the subject of recent theoretical analysis $[\underline{11}, \underline{12}]$.
In Section $\underline{2}$ we identify all possible linear, circular, and elliptical states in three dimensions and derive expressions for their respective GJVs. In Section 3 we consider the subset of 3-D PSs obtained by the superposition of three mutually orthogonal $x, y$, and $z$ oscillating components of the electric field of equal amplitudes as functions of their relative phases. Section 4 gives an example of a $3 \times 3$ Jones matrix that relates the GJVs of the incident and evanescent refracted light in total internal reflection. Finally, Section 5 gives a brief summary of the paper.

\section{GENERALIZED JONES VECTORS OF 3-D LINEAR, CIRCULAR, AND ELLIPTICAL POLARIZATION STATES}

\section{A. Linear States}

Figure 1 shows a right-handed $x y z$ Cartesian coordinate system with origin at an arbitrary point in the optical field and a unit sphere of radius $r=1$. Each linear polarization (LP) state is uniquely specified by a radial line through the origin along which the electric field $\vec{E}$ is oscillating. A unit vector $\hat{n}$ along the vibration direction is determined by the zenith and azimuth angles $(\theta, \phi)$ of spherical coordinates. Apart from a complex multiplier $a e^{j \delta}$ that accounts for an overall amplitude and absolute phase, the Cartesian GJV of a LP state is given by

$$
\begin{aligned}
\boldsymbol{E}_{\mathrm{LP}} & =\left[\begin{array}{lll}
E_{x} & E_{y} & E_{z}
\end{array}\right]^{t}=\hat{n} \\
& =\left[\begin{array}{lll}
\sin \theta \cos \phi & \sin \theta \sin \phi & \cos \theta
\end{array}\right]^{t} .
\end{aligned}
$$

Recall that the time-dependent electric field is given by $\operatorname{Re}\left[a e^{j \delta} E_{\mathrm{LP}} e^{j \omega t}\right]$. Also note that replacing $(\theta, \phi)$ by $(\pi-\theta, \phi+$ $\pi)$ in the right-hand side of Eq. (1) generates $-\boldsymbol{E}_{\mathrm{LP}}$, which does not represent a new LP state. Hence, all possible 3-D LP states 
are obtained if $\theta, \phi$ are limited to the ranges $0 \leq \theta \leq \pi / 2$, $0 \leq \phi \leq 2 \pi$. Stated differently, there are as many distinct 3-D LP states as there are radial lines from the origin to all points on the surface of the upper half of the unit sphere $(z \geq 0)$ inclusive of the equator. By contrast, there are as many two-dimensional (2-D) LP states as there are points on a semicircle or, equivalently, the equator of the Poincaré sphere.

\section{B. Circular States}

With reference to Fig. 1, a 3-D circular polarization (CP) state is defined by a great circle (GC) whose unit normal is $\hat{n}(\theta, \phi)$. The sense of rotation (handedness) of the electric field around the GC is linked to the unit normal to the orbital plane $\hat{n} \equiv \hat{z}^{\prime}$ by the right-hand rule. Therefore, unit vectors $\hat{n}$ and $-\hat{n}$ identify orthogonal (oppositely handed) CP states that share the same orbit. It becomes apparent that there are as many distinct 3-D CP states as there are points on the surface of the unit sphere, i.e., as many as all possible 2-D PSs on the Poincaré sphere of which only two represent circular states. Note that the number of 3-D CP states is double the number of 3-D LP states. By contrast, there are only two CP states versus an infinite number of LP states in two dimensions.

To determine the GJV of a CP state, we define a reference coordinate axes $x^{\prime} y^{\prime}$ in the orbital plane as follows. The $x^{\prime}$ axis is the line of intersection of the GC with the equator (perpendicular to the $\phi=$ constant plane of $\hat{\boldsymbol{z}}^{\prime}, \hat{z}$ ) and is inclined with respect to the $x$ axis by an angle $\phi^{\prime}=\phi-90^{\circ}$. The unit vector $\hat{x}^{\prime}$ along the $x^{\prime}$ axis is given by

$$
\hat{x}^{\prime}=(\sin \phi) \hat{x}-(\cos \phi) \hat{y} .
$$

The orthogonal unit vector $\hat{y}^{\prime}$ in the orbital plane is obtained by the cross product $\hat{n} \times \hat{x}^{\prime}$. From Eqs. (1) and (2), one obtains

$$
\hat{y}^{\prime}=(\cos \theta \cos \phi) \hat{x}+(\cos \theta \sin \phi) \hat{y}-(\sin \theta) \hat{z} .
$$

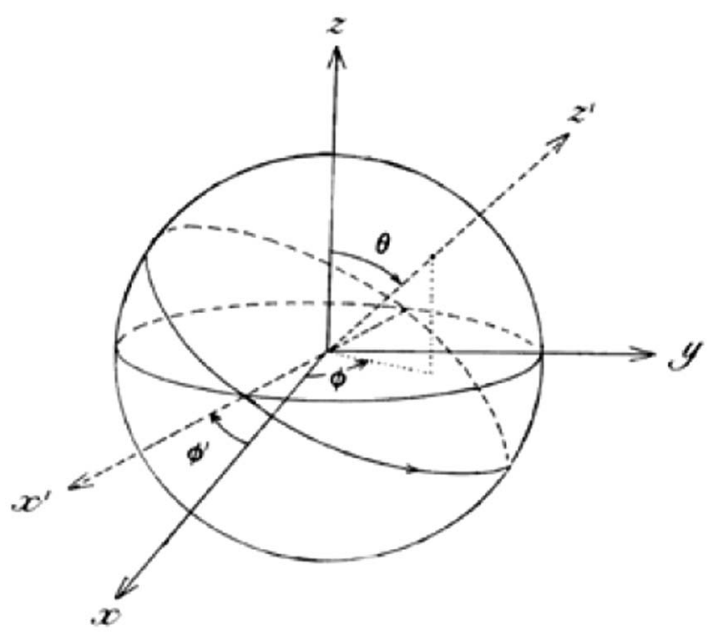

Fig. 1. Right-handed $x y z$ Cartesian coordinate system and the unit sphere of radius $r=1$. A LP state is specified by a radial line through the origin with the zenith and azimuth angles $(\theta, \phi)$ of spherical coordinates. A CP state is represented by a great circle with a normal unit vector $\hat{z}^{\prime}=\hat{n}(\theta, \phi)$ and sense of rotation related to $\hat{n}$ by the righthand rule. An EP state is specified by the unit vector $\hat{z}^{\prime}=\hat{n}(\theta, \phi)$ normal to the $x^{\prime} y^{\prime}$ orbital plane and the orientation and ellipticity angles $\psi, \varepsilon$ of the ellipse (not shown) in that plane.
The time-dependent electric field that represents a CP state with unit normal $\hat{n}$ is given by $(\cos \omega t) \hat{x}^{\prime}+(\sin \omega t) \hat{y}^{\prime}$; the corresponding complex vector in the $x^{\prime} y^{\prime}$ plane is

$$
\boldsymbol{E}_{\mathrm{CP}}=\hat{x}^{\prime}-j \hat{y}^{\prime}
$$

Upon substitution of the unit vectors $\hat{x}^{\prime}, \hat{y}^{\prime}$ from Eqs. (2) and (3) into Eq. (4), one gets

$$
\begin{aligned}
\boldsymbol{E}_{\mathrm{CP}} & =\left[\begin{array}{lll}
E_{x} & E_{y} & E_{z}
\end{array}\right]^{t} \\
& =\left[\begin{array}{lll}
(\sin \phi-j \cos \theta \cos \phi) & -(\cos \phi+j \cos \theta \sin \phi) & j \sin \theta
\end{array}\right]^{t} .
\end{aligned}
$$

Apart from a complex multiplier $a e^{j \delta}$ that adjusts the overall amplitude and phase, Eq. (ㅁ) gives the GJV of a CP state with unit normal $\hat{n}(\theta, \phi)$. For example, the GJV of the CP in the $x y$ plane with $\hat{n}=\hat{z}$ is obtained by substituting $\theta=0$ in Eq. (5) to give $\boldsymbol{E}_{\mathrm{CP}}=\exp [j(\phi-\pi / 2)]\left[\begin{array}{lll}1 & -j & 0\end{array}\right]^{t}$, where $\phi$ is an arbitrary phase angle.

\section{Elliptical States}

An elliptic polarization (EP) state in the $x^{\prime} y^{\prime}$ plane with unit normal $\hat{z}^{\prime}=\hat{n}(\theta, \phi)$, major-axis orientation angle $\psi(0 \leq \psi<\pi$ measured from the $x^{\prime}$ direction around the $z^{\prime}$ axis), and ellipticity angle $\varepsilon(0<\varepsilon=\arctan (b / a)<\pi / 4$, where $a$ and $b$ are the semimajor and semiminor axes of the polarization ellipse) is described by the complex vector

$$
\begin{aligned}
\boldsymbol{E}_{\mathrm{EP}}= & (\cos \psi \cos \varepsilon+j \sin \psi \sin \varepsilon) \hat{x}^{\prime} \\
& +(\sin \psi \cos \varepsilon-j \cos \psi \sin \varepsilon) \hat{y}^{\prime}
\end{aligned}
$$

Equation (6) follows from Eq. (1.75) of [3] by replacing $\theta$ by $\psi$ and $j$ by $-\bar{j}$. Upon substitution of the unit vectors $\hat{x}^{\prime}, \hat{y}^{\prime}$ from Eqs. (2) and (ㅁ) into Eq. (ㅁ), we obtain

$$
\begin{aligned}
\boldsymbol{E}_{\mathrm{EP}}= & {\left[\begin{array}{lll}
E_{x} & E_{y} & E_{z}
\end{array}\right]^{t} ; } \\
E_{x}= & \cos \varepsilon(\sin \phi \cos \psi+\cos \theta \cos \phi \sin \psi) \\
& +j \sin \varepsilon(\sin \phi \sin \psi-\cos \theta \cos \phi \cos \psi), \\
E_{y}= & -\cos \varepsilon(\cos \phi \cos \psi-\cos \theta \sin \phi \sin \psi) \\
& -j \sin \varepsilon(\cos \phi \sin \psi+\cos \theta \sin \phi \cos \psi), \\
E_{z}= & -\sin \theta(\cos \varepsilon \sin \psi-j \sin \varepsilon \cos \psi) .
\end{aligned}
$$

Apart from a complex multiplier $a e^{j \delta}$ that accounts for an overall amplitude and phase, Eqs. (7) give the GJV of an EP state with unit normal $\hat{n}(\theta, \phi)$ and orientation and ellipticity angles $(\psi, \varepsilon)$ of the polarization ellipse in its orbital plane.

Equations (7) are important in that they provide the 3-D GJV in terms of the four geometric parameters $(\theta, \phi ; \psi, \varepsilon)$ of the general 3-D EP state. There is one-to-one correspondence between distinct 3-D PSs of a monochromatic light field and points in the region of the four-dimensional parameter space of $(\theta, \phi ; \psi, \varepsilon)$, which is specified by the limits that have been set for these variables. Also, note that CP states are recovered from Eqs. (7) by setting $\varepsilon=\pi / 4$. Likewise, all LP states (of variable orientation $\psi$ in the $x^{\prime} y^{\prime}$ plane) that are orthogonal to a given LP state or direction $\hat{z}^{\prime}=\hat{n}(\theta, \phi)$ are obtained by substituting $\varepsilon=0$ in Eqs. (7): 


$$
\begin{aligned}
& E_{x}=\sin \phi \cos \psi+\cos \theta \cos \phi \sin \psi, \\
& E_{y}=-\cos \phi \cos \psi+\cos \theta \sin \phi \sin \psi, \\
& E_{z}=-\sin \theta \sin \psi .
\end{aligned}
$$

The reader can readily verify that the dot product of the two vectors in Eqs. (1) and (8) is zero.

The polarization states of far-field scattering are two dimensional and transverse to the scattering direction. If $z^{\prime}$ in Fig. 1 represents a given scattering direction, then Eq. (6) represents all possible 2-D PSs in the transverse $x^{\prime} y^{\prime}$ plane.

\section{POLARIZATION STATES OBTAINED BY THE SUPERPOSITION OF THREE MUTUALLY ORTHOGONAL LINEAR COMPONENTS OF EQUAL AMPLITUDE}

Interest in the generation and possible application of such PSs has been expressed recently [4]. There is no loss of generality if we take all amplitudes $=1$ and assign zero phase to the $x$ component. Therefore, the time-dependent electric-field components are written as

$$
\begin{aligned}
& E_{x t}=\cos (\omega t), \quad E_{y t}=\cos \left(\omega t+\delta_{y}\right), \\
& E_{z t}=\cos \left(\omega t+\delta_{z}\right) .
\end{aligned}
$$

The phase angles $\delta_{y}, \delta_{z}$ assume values in the ranges $-\pi \leq \delta_{y} \leq \pi,-\pi \leq \delta_{z} \leq \pi$. The squared magnitude of the electric vector $\vec{E}$ of Eqs. (9) is

$$
\begin{aligned}
|\vec{E}|^{2} & =\frac{3}{2}+A \cos (2 \omega t)+B \sin (2 \omega t), \\
A & =\frac{1}{2}\left[1+\cos \left(2 \delta_{y}\right)+\cos \left(2 \delta_{z}\right)\right], \\
B & =-\frac{1}{2}\left[\sin \left(2 \delta_{y}\right)+\sin \left(2 \delta_{z}\right)\right] .
\end{aligned}
$$

Alternatively, Eq. (10) can be rewritten as

$$
\begin{aligned}
|\vec{E}|^{2} & =1.5+C \cos (2 \omega t-\gamma), \quad C=\sqrt{A^{2}+B^{2}}, \\
\gamma & =\arctan (B / A) .
\end{aligned}
$$

\section{A. 3-D Polarization States of Equal Ellipticity}

From Eqs. (12), the squared semimajor and semiminor axes of the polarization ellipse are given by

$$
|\vec{E}|_{\max }^{2}=1.5+C, \quad|\vec{E}|_{\min }^{2}=1.5-C .
$$

The ellipticity or axial ratio $e=b / a$ of the 3 -D polarization ellipse is determined by $C$ only:

$$
e=|\vec{E}|_{\min } /|\vec{E}|_{\max }=[(1.5-C) /(1.5+C)]^{1 / 2} .
$$

From Eqs. (11) and (12), $C$ is obtained as a function of the phase angles $\delta_{y}, \delta_{z}$ :

$$
2 C^{2}=1.5+\cos \left(2 \delta_{y}\right)+\cos \left(2 \delta_{z}\right)+\cos \left(2 \delta_{y}-2 \delta_{z}\right) .
$$

LP states $\left(|\vec{E}|_{\min }=0\right)$ correspond to $C=1.5$, whereas CP states $\left(|\vec{E}|_{\min }=|\vec{E}|_{\max }\right)$ correspond to $C=0$ as is evident

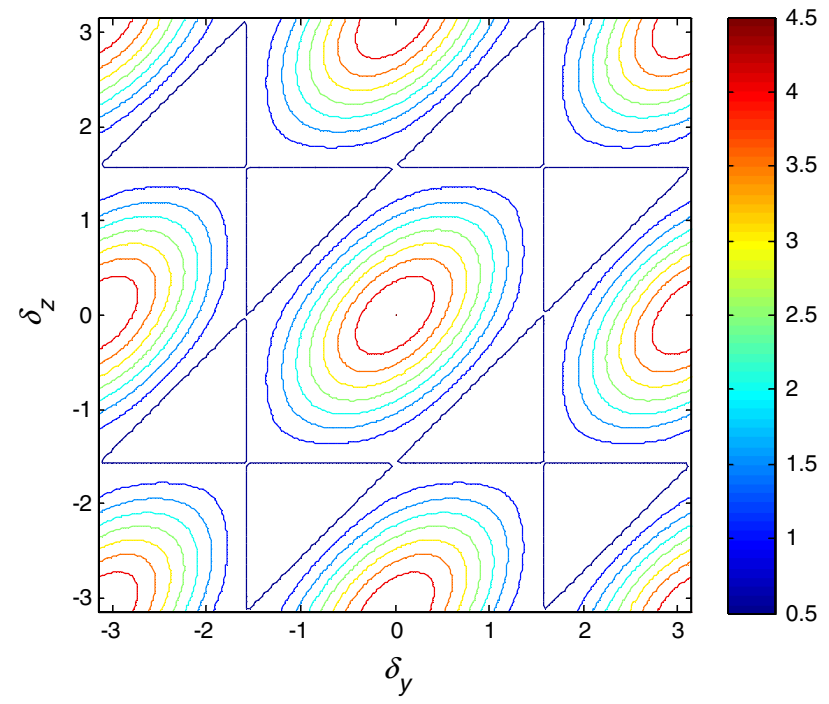

Fig. 2. (Color online) Contours of equal ellipticity ( $e=$ constant) in the $\delta_{y} \delta_{z}$ phase space for 3-D polarization states generated by the superposition of three mutually orthogonal linear components of equal amplitude [Eqs. (9)]. This family of contours corresponds to values of $2 C^{2}$ [Eq. (15)] in the range from 0.5 to 4.5 .

from Eqs. (13). Therefore, $2 C^{2}$ of Eq. (15) occupies the full range from 0 to 4.5 .

Figure 2 shows constant-e contours in the $\delta_{y} \delta_{z}$ plane for values of $2 C^{2}$ from 0.5 to 4.5 . The center point of the diagram $\left(\delta_{y}=\delta_{z}=0\right.$ ) represents the LP state $L_{1}$ along the unit vector $\hat{n}_{1}=(1,1,1) / \sqrt{3}$, which is the center line of the first octant of the $x y z$ coordinate system. Because of fourfold symmetry around the $z$ axis, shifting $\delta_{y}, \delta_{z}$ or both by $\pm \pi$ leads to only three additional distinct LP states $L_{2}, L_{3}$, and $L_{4}$, with unit vectors $\hat{n}_{2}=(-1,1,1) / \sqrt{3}, \quad \hat{n}_{3}=(-1,-1,1) / \sqrt{3}$, and $\hat{n}_{4}=$ $(1,-1,1) / \sqrt{3}$ in the second, third, and fourth octants $(z>0)$ of the $x y z 3$-D space, respectively.

In Fig. 2 the set of straight lines $\delta_{y}= \pm \pi / 2, \delta_{z}= \pm \pi / 2$, $\delta_{z}=\delta_{y} \pm \pi / 2$, and $\delta_{z}=\delta_{y} \pm 3 \pi / 2$ are loci of all 3-D EP states with $e=1 / \sqrt{2}=0.707$ and $2 C^{2}=0.5$. The projections of

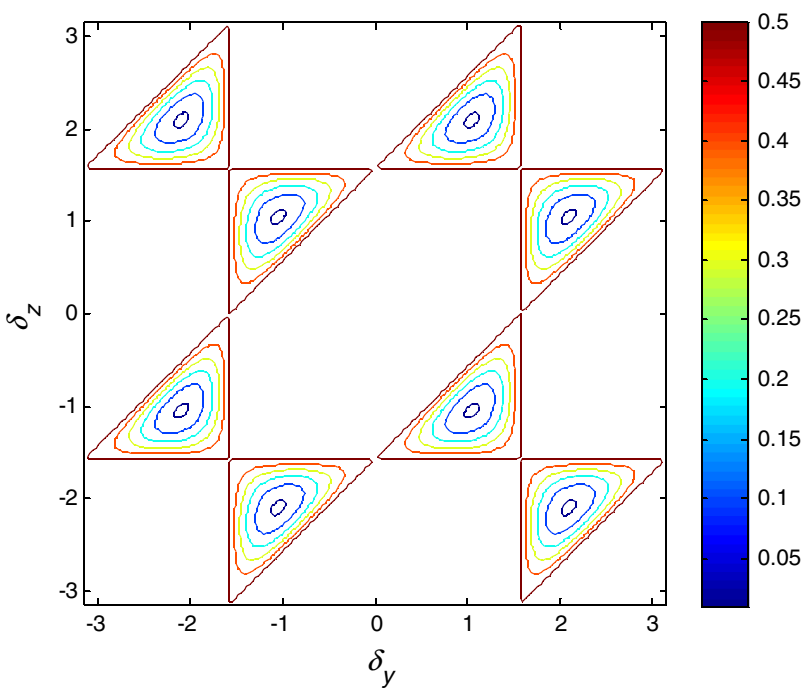

Fig. 3. (Color online) Continuation of the $e=$ constant contours of Fig. 2 for values of $2 C^{2}$ [Eq. (15)] from 0 to 0.5 . This family of contours represents near-circular polarization states with $0.707 \leq e \leq 1$. The "bull's eyes" in the triangular regions represent eight distinct 3-D CP states $(e=1)$ 
these 3 -D EP states in the $x y, x z$, and $y z$ coordinate planes represent 2-D CP states.

Figure 3 is a continuation of Fig. 2 for values of $2 C^{2}$ from 0 to 0.5 . These contours represent near-circular, constant- $e, 3-\mathrm{D}$ PSs with $0.707 \leq e \leq 1$. The "bull's eyes" in the triangular regions represent eight distinct 3 -D $\mathrm{CP}$ states $(e=1)$ that correspond to $\left(\delta_{y}, \delta_{z}\right)= \pm(\pi / 3,-\pi / 3), \quad \pm(2 \pi / 3,-2 \pi / 3)$, $\pm(\pi / 3,2 \pi / 3)$, and $\pm(2 \pi / 3, \pi / 3)$.

The 3-D CP state $C 1$ with unit normal $\hat{n}_{1}=(1,1,1) / \sqrt{3}$ corresponds to $\left(\delta_{y}, \delta_{z}\right)=(-2 \pi / 3,2 \pi / 3)=\left(-120^{\circ}, 120^{\circ}\right)$. For this CP state, the triad of phasors $E_{x}, E_{y}$, and $E_{z}$ that represent the three oscillating Cartesian components is a balanced (zerosum) three-phase set in the complex plane, as shown in Fig. 4. This is reminiscent of the balanced set of ac voltages in threephase power systems [13]. Note that, if the phasors $E_{y}$ and $E_{z}$ are interchanged, the CP state $C 2$ of opposite handedness and unit normal $\hat{n}_{2}=-\hat{n}_{1}$ is obtained. In Appendix A we show how these CP states can be obtained directly from Eq. (5).

\section{B. Unit Vector Normal to the Plane of the Polarization Ellipse in Terms of the Phase Angles of the Oscillating $y$ and $z$ Components}

Evaluation of the instantaneous electric vector $\vec{E}$ of Eqs. (9) at $\omega t=0, \pi / 2$ yields

$$
\vec{E}_{1}=\left(1, \cos \delta_{y}, \cos \delta_{z}\right), \quad \vec{E}_{2}=\left(0,-\sin \delta_{y},-\sin \delta_{z}\right) .
$$

The unit vector $\hat{n}$ normal to the orbital plane of the polarization ellipse is determined by

$$
\hat{n}=\left(\vec{E}_{1} \times \vec{E}_{2}\right) /\left|\vec{E}_{1} \times \vec{E}_{2}\right| .
$$

From Eqs. (16) and (17) we get

$$
\hat{n}=\frac{\sin \left(\delta_{y}-\delta_{z}\right) \hat{x}+\left(\sin \delta_{z}\right) \hat{y}-\left(\sin \delta_{y}\right) \hat{z}}{+\left[\sin ^{2} \delta_{y}+\sin ^{2} \delta_{z}+\sin ^{2}\left(\delta_{y}-\delta_{z}\right)\right]^{1 / 2}} .
$$

The inclination angle $\alpha$ of the unit normal $\hat{n}$ with respect to the positive $x$ axis is given by

$$
\begin{aligned}
\cos \alpha & =\hat{n} . \hat{x} \\
& =\sin \left(\delta_{y}-\delta_{z}\right)\left[\sin ^{2} \delta_{y}+\sin ^{2} \delta_{z}+\sin ^{2}\left(\delta_{y}-\delta_{z}\right)\right]^{-1 / 2} .
\end{aligned}
$$

Figure $\underline{5}$ shows constant- $\alpha$ contours in the $\delta_{y} \delta_{z}$ plane for discrete values of $\cos \alpha$ from -0.8 to 0.8 in steps of 0.1 . To facilitate understanding of this figure, we provide the following details.

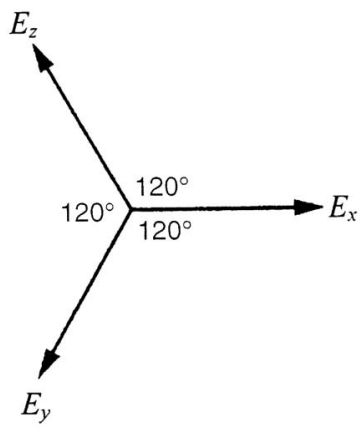

Fig. 4. Balanced (zero-sum) set of three phasors $E_{x}, E_{y}$, and $E_{z}$ [Eqs. (A2)] that generate a CP state with unit normal $\hat{n}=(1,1,1) / \sqrt{3}$.

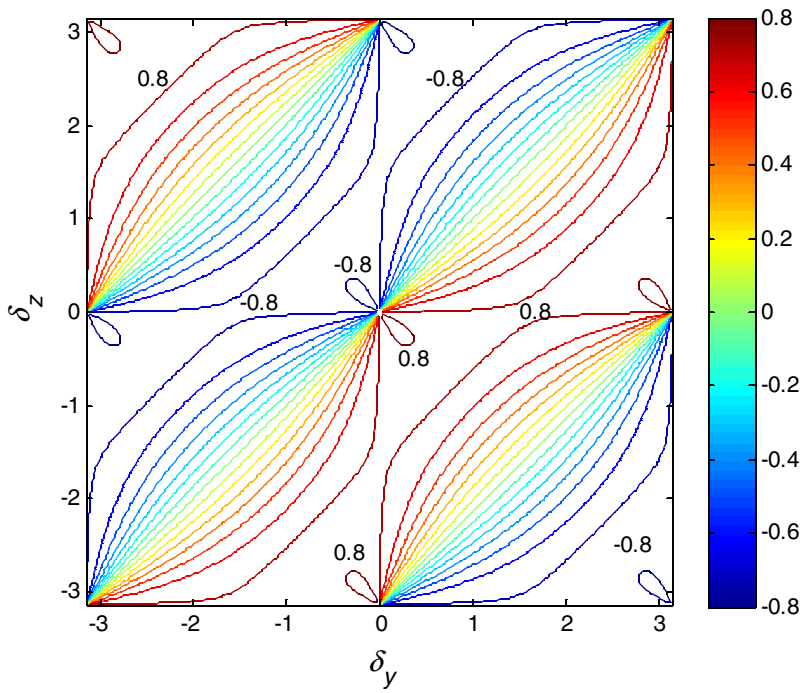

Fig. 5. (Color online) Contours of constant inclination angle $\alpha$ (between the normal to the plane of the polarization ellipse and the positive $x$ axis) in the $\delta_{y} \delta_{z}$ plane for values of $\cos \alpha$ from -0.8 to 0.8 in steps of 0.1 . (See text for detail.)

1. For LP states $\left(\delta_{y}, \delta_{z}\right)=(0,0),(0, \pm \pi),( \pm \pi, 0)$, and $( \pm \pi, \pm \pi)$, the unit vector $\hat{n}$ and $\cos \alpha$ are indeterminate according to Eq. (18), which reduces to $\hat{n}=\overrightarrow{0} / 0$.

2. Equation (19) shows that $\cos \alpha=0, \alpha=90^{\circ}$ (i.e., $\hat{n}$ is in the $y z$ coordinate plane) when $\delta_{y}-\delta_{z}=0, \pm \pi$. The corresponding PSs are represented by the three diagonal lines with slope $=1$.

3. When $\delta_{y}=0$, Eq. (19) gives $\cos \alpha=\mp 1 / \sqrt{2}=\mp 0.707$ for $\delta_{z}>0, \delta_{z}<0$, respectively. Likewise, when $\delta_{z}=0, \cos \alpha=$ $\pm 1 / \sqrt{2}= \pm 0.707$ for $\delta_{y}>0, \delta_{y}<0$, respectively.

4. The cross-diagonal straight line $\delta_{z}=-\delta_{y}$ with slope $=$ -1 is a line of the gradient of $\cos \alpha$. Along this line, Eq. (19) simplifies to

$$
\cos \alpha=\cos \delta_{y} / \sqrt{0.5+\cos ^{2} \delta_{y}} .
$$

Limiting values, $\cos \alpha= \pm \sqrt{2 / 3}= \pm 0.8165$, are obtained when $\cos \delta_{y}= \pm 1, \delta_{y}=0, \pm \pi$. This leads to the interesting conclusion that the smallest possible inclination angle of the unit normal $\hat{n}$ with respect to the positive or negative $x$ axis is $35.2644^{\circ}$.

\section{GENERALIZED $3 \times 3$ JONES MATRICES}

By straightforward extension of the $2 \times 2$ Jones calculus [3], elastic scattering, e.g., by a nano-object in the near field, is characterized by the linear transformation

$$
\boldsymbol{E}_{s}=\boldsymbol{T} \boldsymbol{E}_{i}
$$

in which $\boldsymbol{E}_{i}$ and $\boldsymbol{E}_{s}$ are the $3 \times 1$ GJVs of the incident and scattered waves and $\boldsymbol{T}$ is the $3 \times 3$ generalized Jones matrix (GJM) of the scatterer at a given frequency and for given incidence and scattering directions.

There is a scarcity of theoretical or experimental examples in which the $3 \times 3$ generalized Jones calculus, implied by Eq. (21), has been put to use. Therefore, it will suffice here to take the case of evanescent refraction that accompanies total internal reflection (TIR) at the planar interface between 


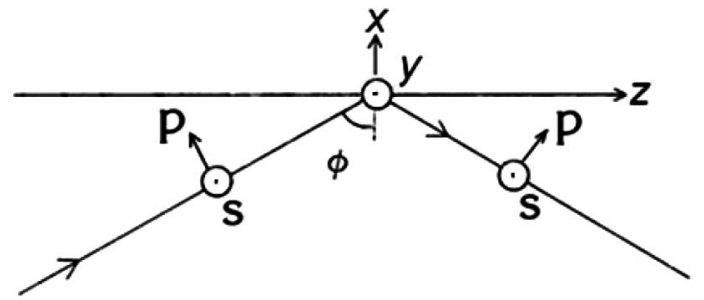

Fig. 6. Geometry of TIR at an angle $\phi$ at a dielectric-dielectric interface, the reference $x y z$ coordinate system, and the $p$ and $s$ linear polarizations of incident and reflected light.

two dielectric media [7-9]. For an arbitrary polarization of the incident plane wave in the denser medium (with both $p$ and $s$ components present), the polarization of the evanescent field in the rarer medium is three dimensional. Here we adopt the $e^{j \omega t}$ time dependence, the Nebraska-Muller conventions $[3,14]$, and the $x y z$ coordinate system shown in Fig. $\underline{6}$. The $\mathrm{GJVs}$ of the incident $(i)$ and evanescent $(e)$ waves are written as

$$
\begin{aligned}
& \boldsymbol{E}_{i}=\left[\begin{array}{lll}
E_{i x} & E_{i y} & E_{i z}
\end{array}\right]^{t} \\
& =\left[\begin{array}{lll}
(\sin \phi) E_{i p} & E_{i s} & (-\cos \phi) E_{i p}
\end{array}\right]^{t}, \\
& \boldsymbol{E}_{e}=\left[\begin{array}{lll}
E_{e x} & E_{e y} & E_{e z}
\end{array}\right]^{t} .
\end{aligned}
$$

In Eq. (22), $E_{i p}$ and $E_{i s}$ are the phasors that represent the $p$ and $s$-polarized components of incident light and $\phi$ is the angle of incidence that exceeds the critical angle of TIR. Application of boundary conditions to the normal $(x)$ and tangential ( $y$ and $z)$ components of the electric field at the dielectric-dielectric interface leads to a diagonal $3 \times 3 \mathrm{GJM}$ with nonzero elements given by

$$
\begin{aligned}
& T_{11}=N^{2}\left(1+r_{p}\right), \quad T_{22}=\left(1+r_{s}\right), \\
& T_{33}=\left(1-r_{p}\right) .
\end{aligned}
$$

In Eqs. (24), $N$ is the high-to-low refractive-index ratio of the two dielectrics, and $r_{p}, r_{s}$ are the interface Fresnel coefficients of TIR that are pure phase factors:

$$
r_{p}=\exp \left(j \delta_{p}\right), \quad r_{s}=\exp \left(j \delta_{s}\right) .
$$

Expressions for $\delta_{p}, \delta_{s}$ in terms of $N, \phi$ are available elsewhere (e.g., [15]).

\section{SUMMARY}

GJVs that describe all possible 3-D linear, circular, and elliptic polarization states of monochromatic light in the near field are given by Eqs. (1), (5), and (7), respectively, in terms of four angles $(\theta, \phi ; \psi, \bar{\varepsilon})$ that describe the 3 -D vibration of the electric field. The subset of 3-D polarization states that are obtained by the superposition of three mutually orthogonal linear components of equal amplitude are also considered as a function of their relative phase angles $\delta_{y}, \delta_{z}\left(\delta_{x}=0\right)$. Contours of constant ellipticity $e$ in the 2-D $\delta_{y}, \delta_{z}$ phase space are shown in Figs. 2 and 3 . Furthermore, contours of constant inclination angle $\alpha$ of the normal to the plane of the polarization ellipse with respect to the $x$ axis appear in Fig. 4 . A $3 \times 3$ generalized Jones calculus is briefly introduced in Section $\underline{4}$ with reference to the $3-\mathrm{D}$ evanescent field generated in TIR. We hope this paper will encourage further work on the development and application of the $3 \times 3$ Jones calculus in near-field optics and electromagnetics.

Potential applications of 3-D polarization states include polarization-dependent interaction of light with arbitrarily oriented anisotropic nanoparticles (e.g., Au nanorods), fluorescent imaging, single-molecule detection, and optical microscopy, as has been suggested recently [푸, 16$]$.

\section{APPENDIX A}

The $x, y$, and $z$ components of the electric vector that produce the CP state with unit normal $\hat{n}=(1,1,1) / \sqrt{3}$ are derived from Eq. (5) by substituting $\theta=\cos ^{-1}(1 / \sqrt{3}), \phi=45^{\circ}$ :

$E_{x}=\sqrt{2 / 3} \exp (-j \pi / 6), \quad E_{y}=\sqrt{2 / 3} \exp (-j 5 \pi / 6)$,

$E_{z}=\sqrt{2 / 3} \exp (j \pi / 2)$.

Multiplication of the three phasors of Eqs. (A1) by $\sqrt{3 / 2} \exp (j \pi / 6)$ gives

$$
E_{x}=1, \quad E_{y}=\exp (-j 2 \pi / 3), \quad E_{z}=\exp (j 2 \pi / 3),
$$

so that the standard balanced 3-phasor set shown in Fig. $\underline{4}$ is recovered.

\section{REFERENCES}

1. J. T. Verdeyen, Laser Electronics (Prentice Hall, 1995), Sec. 3.2.

2. H. Poincaré, Théorie Mathématique de la Lumière (GauthiersVillares, 1892).

3. R. M. A. Azzam and N. M. Bashara, Ellipsometry and Polarized Light (North-Holland, 1987), Sec. 1.3.

4. H. Kang, B. Jia, and M. Gu, "Polarization characterization in the focal volume of high numerical aperture objectives," Opt. Express 18, 10813-10821 (2010).

5. Q. Zhan, "Cylindrical vector beams: from mathematical concepts to applications," Adv. Opt. Photon. 1, 1-57 (2009).

6. H. A. Haus, Waves and Fields in Optoelectronics (Prentice Hall, 1984), Sec. 6.3.

7. F. de Fornel, Evanescent Waves (Springer, 2001).

8. L. Józefowski, J. Fiutowski, T. Kawalec, and H.-G. Rubahn, "Direct measurement of the evanescent-wave polarization state," J. Opt. Soc. Am. B 24, 624-628 (2007).

9. L. Novotny and B. Hecht, Principles of Nano-Optics (Cambridge University, 2006).

10. A. T. Adams and E. Mendelovich, "The near-field polarization ellipse," IEEE Trans. Antennas Propag. AP-21, 124-126 (1973).

11. C. Brosseau and A. Dogariu, "Symmetry properties and polarization descriptors for an arbitrary electromagnetic wavefield," in Vol. 49 of Progress in Optics, E. Wolf, ed. (Elsevier, 2006), Chap. 4.

12. A. Norrman, T. Setälä, and A. T. Friberg, "Partial coherence and partial polarization in random evanescent fields on lossless interfaces," J. Opt. Soc. Am. A 28, 391-400 (2011).

13. R. C. Dorf and J. A. Svoboda, Introduction to Electric Circuits, 4th ed. (Wiley, 1999), Sec. 12.3.

14. R. H. Muller, "Definitions and conventions in ellipsometry," Surf. Sci. 16, 14-33 (1969).

15. R. M. A. Azzam, "Phase shifts that accompany total internal reflection at a dielectric-dielectric interface," J. Opt. Soc. Am. A 21, 1559-1563 (2004).

16. W. Chen and Q. Zhan, "Three dimensional polarization control in 4Pi microscopy," Opt. Commun. 284, 52-56 (2011). 